\title{
Natural realizations of sparsity matroids
}

\author{
Ileana Streinu * \\ Computer Science Department, Ford Hall, Smith College \\ Northampton, MA 01063, USA \\ Louis Theran ${ }^{\dagger}$ \\ Mathematics Department, Temple University \\ 1805 North Broad Street, Philadelphia, PA 19122, USA
}

Received 5 July 2010, accepted 16 December 2010, published online 19 March 2011

\begin{abstract}
A hypergraph $G$ with $n$ vertices and $m$ hyperedges with $d$ endpoints each is $(k, \ell)$ sparse if for all sub-hypergraphs $G^{\prime}$ on $n^{\prime}$ vertices and $m^{\prime}$ edges, $m^{\prime} \leq k n^{\prime}-\ell$. For integers $k$ and $\ell$ satisfying $0 \leq \ell \leq d k-1$, this is known to be a linearly representable matroidal family.

Motivated by problems in rigidity theory, we give a new linear representation theorem for the $(k, \ell)$-sparse hypergraphs that is natural; i.e., the representing matrix captures the vertex-edge incidence structure of the underlying hypergraph $G$.
\end{abstract}

Keywords: Matroids, combinatorial rigidity, sparse graphs and hypergraphs.

Math. Subj. Class.: 52C25, 05B35, 05C65, 68R10

\section{Introduction}

Let $G$ be a d-uniform hypergraph; i.e., $G=(V, E)$, where $V$ is a finite set of $n$ vertices and $E$ is a multi-set of $m$ hyperedges, which each have $d$ distinct endpoints. We define $G$ to be $(k, \ell)$-sparse if, for fixed integer parameters $k$ and $\ell$, any non-empty sub-hypergraph $G^{\prime}$ of $G$ on $n^{\prime}$ vertices and $m^{\prime}$ hyperedges satisfies the relation $m^{\prime} \leq k n^{\prime}-\ell ;$ if, in addition $m=k n-\ell$, then $G$ is $(k, \ell)$-tight.

For a fixed $n$, and integer parameters $k, \ell$, and $d$ satisfying $0 \leq \ell \leq d k-1$, the family of $(k, \ell)$-tight $d$-uniform hypergraphs on $n$ vertices form the bases of a matroid [21], which we define to be the $(k, \ell)$-sparsity-matroid. The topic of this paper is linear representations of the $(k, \ell)$-sparsity-matroids with a specific form.

\footnotetext{
* Supported by grants NSF CCF-1016988, CCF-0728783 and a DARPA 23 Mathematical Challenges grant.

$\dagger$ This author's PhD research was funded by grant NSF CCF-0728783 to Ileana Streinu, with preparation supported by CDI-I grant DMR 0835586 to Igor Rivin and M. M. J. Treacy.

E-mail addresses: istreinu@smith.edu (Ileana Streinu), theran@temple.edu (Louis Theran)
} 
Main Theorem. Our main result is the following. Detailed definitions of $(k, \ell)$-sparse hypergraphs are given in Section 2; detailed definitions of linear representations are given in Section 3.

Theorem 1.1 (Natural Realizations). Let $k$, $\ell$, and $d$ be integer parameters satisfying the inequality $0 \leq \ell \leq k d-1$. Then, for sufficiently large $n$, the $(k, \ell)$-sparsity-matroid of $d$-uniform hypergraphs on $n$ vertices is representable by a matrix $\mathbf{M}$ with:

- Real entries

- $k$ columns corresponding to each vertex (for a total of $k n$ )

- One row for each hyperedge e

- In the row corresponding to each edge e, the only non-zero entries appear in columns corresponding to endpoints of $e$

Novelty. As a comparison, standard matroidal constructions imply that there is a linear representation that is $m \times k n$ for all the allowed values of $k, \ell$ and $d$. For $d=2, \ell \leq k$, the $(k, \ell)$-sparsity-matroid is characterized as the matroid union of $\ell$ copies of the standard graphic matroid and $(k-\ell)$ copies of the bicycle matroid, so the desired representation follows from the Matroid Union Theorem [2, Section 7.6] for linearly representable matroids.

Theorem 1.1, in contrast, applies to the entire matroidal range of parameters $k, \ell$, and $d$. In particular, it applies in the so-called upper range in which $\ell>k$. In the upper range, no reduction to matroid unions are known, so proofs based on the Matroid Union Theorem do not apply.

Motivation. Our motivation for this work comes from rigidity theory, which is the study of structures defined by geometric constraints. Examples include: bar-joint frameworks, which are structures made of fixed-length bars connected by universal joints, with full rotational freedom; and body-bar frameworks, which are made of rigid bodies connected by fixed length bars attached to universal joints. A framework is rigid if the only allowed continuous motions that preserve the lengths and connectivity of the bars are rigid motions of Euclidean space.

In both cases, the formal description of the framework is given in two parts: a graph $G$, defining the combinatorics of the framework; geometric data, specifying the lengths of the bars, and their attachment points on the bodies. Rigidity is a difficult property to establish in all cases, the with best known algorithms relying on exponential-time Gröbner basis computations. However, for generic geometric data (and almost all lengths are generic, see [16] for a detailed discussion), rigidity properties can be determined from the combinatorics of the framework alone, as shown by the following two landmark theorems:

Theorem 1.2 (Maxwell-Laman Theorem: Generic planar bar-joint rigidity [7, 13]). $A$ generic bar-joint framework in $\mathbb{R}^{2}$ is minimally rigid if and only if its underlying graph $G$ is $(2,3)$-tight.

Theorem 1.3 (Tay's Theorem: Generic body-bar rigidity [17]). A generic body-bar framework in $\mathbb{R}^{d}$ is minimally rigid if and only if its underlying graph $G$ is $(D, D)$-tight, where $D=\left(\begin{array}{c}d+1 \\ 2\end{array}\right)$. 
All known proofs of theorems such as 1.2 and 1.3 proceed via a linearization of the problem called infinitesimal rigidity. The key step in all of these proofs is to prove that a specific matrix, called the rigidity matrix, which arises as the differential of the equations for the length constraints, is, generically, a linear representation of some $(k, \ell)$-sparsity matroid.

The rigidity matrices arising in Theorems 1.2 and 1.3 are specializations of our natural realizations: they have the same pattern of zero and non-zero entries. The present work arises out of the first author's longer-term research project aimed at understanding rigidity "from the combinatorics up" by studying $(k, \ell)$-sparse graphs and their generalizations. Our main Theorem 1.1 and the implied natural realizations occupy an intermediate position in between the rigidity theorems and the combinatorial matroids of $(k, \ell)$-sparse graphs. The natural realizations presented here may be useful as building blocks for a new, more general class of rigidity theorems in the line of Theorems 1.2 and 1.3.

Related work: $(k, \ell)$-sparse graphs. Graphs and hypergraphs defined by hereditary sparsity counts first appeared as an example of matroidal families in the work of Lorea [11]. Whiteley, as part of a project with Neil White, reported in [21, Appendix], studied them from the rigidity perspective. Michael Albertson and Ruth Haas [1] studied $(k, \ell)$-sparse graphs from an extremal perspective as an instance of graphs characterized by "bounding functions."

The results in this paper build upon a sequence of papers by the first author, her students and collaborators: [9] develops the structural and algorithmic theory of $(k, \ell)$-sparse graphs; [15] extends the results of [9] to hypergraphs; [5, 14] give characterizations in terms of decompositions into trees and "map-graphs"; [10] extends the sparsity concept to allow different counts for different types of edges.

Related work: matroid representations. For the specific parameter values $d=2$, $\ell \leq k$, natural realizations of the type presented in Theorem 1.1 may be deduced from the Matroid Union Theorem [2, Section 7.6]; this was done by Whiteley [19], where the realizations for $d=2, \ell=l$ go by the name " $k$-frame." In addition, White and Whiteley [21, Appendix] have shown, using a geometric construction involving picking projective flats in general position and then the Higgs Lift [2, Section 7.5] that all $(k, \ell)$-sparsity matroids for graphs and hypergraphs are linearly representable.

Whiteley [20] proved a very similar result for the special case of $k=1$; he also gave representations for a related class of matroids on bipartite incidence graphs. These matroids have also appeared in the Ph.D. thesis of Audrey Lee-St. John [8] under the name mixed sparsity.

All known rigidity representation theorems [7, 6, 16, 17, 19] provide natural realizations for the specific sparsity parameters involved. However, all these give more specialized representations, arising from geometric considerations, with more specialized proofs. All the arguments having a matroidal flavor seem to rely, in one way or another, on the Matroid Union Theorem, or the explicit determinantal formulas used to prove it.

Related work: rigidity theory. Lovász and Yemini [12] introduced the matroidal perspective to rigidity theory with their proof of the Maxwell-Laman Theorem 1.2 based on an explicit computation of the rank function of the $(2,3)$-sparsity matroid that uses its special relationship with the union of graphic matroids. Whiteley [19] gives a very elegant proof of 
Tay's Theorem 1.3 [17] using the Matroid Union Theorem and geometric observations specific to the body-bar setting. White and Whiteley [18] analyzed the minors of $k$-frames of [19] in detail, describing "pure conditions" that determine the rigidity behavior of body-bar frameworks.

In both [12, 19], as well as in more recently proven Maxwell-Laman-type theorems of Katoh and Tanigawa [6] and the authors' [16], the connection between $(k, \ell)$-sparsity and sparsity-certifying decompositions [14] of the minimally rigid family of graphs appears in an essential way. In contrast, here we only need to employ sparsity itself, yielding a much more general family of realizations. The price for this added generality is that we cannot immediately deduce rigidity results directly from Theorem 1.1.

Organization. Section 2 introduces $(k, \ell)$-sparse hypergraphs and gives the necessary structural properties. Section 3 gives the required background in linear representability of matroids and then the proof of Theorem 1.1. In Section 4, we describe two extensions of Theorem 1.1: to non-uniform $(k, \ell)$-sparse hypergraphs and to $(k, \ell)$-graded-sparse hypergraphs. We conclude in Section 5 with some remarks on the relationship between natural realizations and rigidity.

Notations. A hypergraph $G=(V, E)$ is defined by a finite set $V$ of vertices and a multiset $E$ of hyperedges, which are subsets of $V$; if $e \in E(G)$ is an edge and $v \in e$ is a vertex, then we call $v$ an endpoint of the edge $e$. A hypergraph $G$ is defined to be $d$-uniform if all the edges have $d$ endpoints. Sub-hypergraphs are typically denoted as $G^{\prime}$ with $n^{\prime}$ vertices and $m^{\prime}$ edges; whether they are vertex- or hyperedge-induced will be explicitly states. For $d$-uniform hypergraphs, we use the notation $e_{1}, e_{2}, \ldots, e_{d}$ for the $d$ endpoints of a hyperedge $e \in E(G)$.

Matrices $\mathbf{M}$ are denoted by bold capital letters, vectors $\mathbf{v}$ by bold lowercase letters. The rows of a matrix $\mathbf{M}$ are denoted by $\mathbf{m}_{i}$.

The letters $k, \ell$, and $d$ denote sparsity parameters.

Dedication. This paper is dedicated to the memory of Michael Albertson.

\section{The $(k, \ell)$-sparsity matroid}

Let $(k, \ell, d)$ be a triple of non-negative integers such that $0 \leq \ell \leq d k-1$; we define such a triple as giving matroidal sparsity parameters (this definition is justified below in Proposition 2.1). A $d$-uniform hypergraph $G=(V, E)$ with $n$ vertices and $m$ hyperedges is $(k, \ell)$-sparse if, for all subsets $V^{\prime} \subset V$ of $n^{\prime}$ vertices inducing at least one hyperedge, the subgraph induced by $V^{\prime}$ has $m^{\prime}$ edges with $m^{\prime} \leq k n^{\prime}-\ell$. If, in addition, $m=k n-\ell$, $G$ is $(k, \ell)$-tight. For brevity, we call $(k, \ell)$-tight $d$-uniform hypergraphs $(k, \ell, d)$-graphs.

The starting points for the results of this paper is the matroidal property of $(k, \ell, d)$ graphs. We define $K_{n, d}^{d k-\ell}$ to be the complete $d$-uniform hypergraph on $n$ vertices with $d k-\ell$ copies of each hyperedge.

Proposition 2.1 ([11, 21, 15]). Let $d, k$ and $\ell$ be non-negative integers satisfying $\ell \in$ $[0, d k-1]$. Then the family of $(k, \ell, d)$-graphs on $n$ vertices forms the bases of a matroid on the edges of $K_{n, d}^{d k-\ell}$, for a sufficiently large $n$, depending on $k$, $\ell$, and $d$. 
We define the matroid appearing in Proposition 2.1 to be the $(k, \ell, d)$-sparsity-matroid.

From now on, the parameters $k, \ell$ and $d$ are always matroidal sparsity parameters and $n$ is assumed to be large enough so that Proposition 2.1 holds.

The other fact we need is the following lemma from [15] characterizing the special case of $(k, 0, d)$-graphs. We define an orientation of a hypergraph to be an assignment of a tail to each hyperedge by selecting one of its endpoints (unlike in the graph setting, there is no uniquely defined head).

Lemma 2.2 ([15]). Let $G$ be a d-uniform hypergraph with $n$ vertices and $m=k n$ hyperedges. Then $G$ is a $(k, 0, d)$-graph if and only if there is an orientation such that each vertex is the tail of exactly $k$ hyperedges.

\section{Natural Realizations}

In this section we prove our main theorem:

Theorem 1.1 (Natural Realizations). Let $k, \ell$, and $d$ be integer parameters satisfying the inequality $0 \leq \ell \leq k d-1$. Then, for sufficiently large $n$, the $(k, \ell)$-sparsity-matroid of $d$-uniform hypergraphs on $n$ vertices is representable by a matrix $\mathbf{M}$ with:

- Real entries

- $k$ columns corresponding to each vertex (for a total of $k n$ )

- One row for each hyperedge e

- In the row corresponding to each edge e, the only non-zero entries appear in columns corresponding to endpoints of $e$

Roadmap. This section is structured as follows. We begin by defining generic matrices and then introduce the required background in linear representation of matroids. The proof of Theorem 1.1 then proceeds by starting with the special case of $(k, 0)$-sparse hypergraphs and then reducing to it via a general construction.

The generic rank of a matrix. A generic matrix has as its non-zero entries generic variables, or formal polynomials over $\mathbb{R}$ or $\mathbb{C}$ in generic variables. Its generic rank is given by the largest number $r$ for which $\mathbf{M}$ has an $r \times r$ matrix minor with a determinant that is formally non-zero.

Let $\mathbf{M}$ be a generic matrix in $m$ generic variables $x_{1}, \ldots, x_{m}$, and let $\mathbf{v}=\left(v_{i}\right) \in \mathbb{R}^{m}$ (or $\mathbb{C}^{m}$ ). We define a realization of $\mathbf{M}$ to be the matrix obtained by replacing the variable $x_{i}$ with the corresponding number $v_{i}$. A vector $\mathbf{v}$ is defined to be a generic point if the rank of the associated realization is equal to the generic rank of $\mathbf{M}$; otherwise $\mathbf{v}$ is defined to be a non-generic point.

We will make extensive use of the following well-known facts from algebraic geomety (see, e.g., [3]):

- The rank of a generic matrix $\mathbf{M}$ in $m$ variables is equal to the maximum over $\mathbf{v} \in \mathbb{R}^{m}$ $\left(\mathbb{C}^{m}\right)$ of the rank of all realizations.

- The set of non-generic points of a generic matrix $\mathbf{M}$ is an algebraic subset of $\mathbb{R}^{m}$ $\left(\mathbb{C}^{m}\right)$. 
- The rank of a generic matrix $\mathbf{M}$ in $m$ variables is at least as large as the rank of any specific realization; i.e., generic rank can be established by a single example.

Generic representations of matroids. Let $\mathcal{M}$ be a matroid on ground set $E$. We define a generic matrix $\mathbf{M}$ to be a generic representation of $\mathcal{M}$ if:

- There is a bijection between the rows of $\mathbf{M}$ and the ground set $E$.

- A subset of rows of $\mathbf{M}$ attains the rank of the matrix $\mathbf{M}$ if and only if the corresponding subset of $E$ is a basis of $\mathcal{M}$.

Natural realizations for $(\boldsymbol{k}, \mathbf{0}, \boldsymbol{d})$-graphs. Fix matroidal parameters $k, \ell=0$ and $d$, and let $G$ be a $d$-uniform hypergraph on $n$ vertices and $m$ hyperedges. For a hyperedge $e \in E(G)$ with endpoints $e_{i}, i \in[1, d]$, define the vector $\mathbf{a}_{e_{i}}=\left(a_{e_{i}}^{j}\right)_{j \in[1, k]}$ to have as its entries $k$ generic variables for each of the $d$ endpoints of $e$.

Next, we define the generic matrix $\mathbf{M}_{k, 0, d}(G)$ to have $m$ rows, indexed by the hyperedges of $G$, and $k n$ columns, indexed by the vertices of $G$, with $k$ columns for each vertex. The filling pattern of $\mathbf{M}_{k, 0, d}$ is given as follows:

- If a vertex $i \in V(G)$ is an endpoint of an edge $e$, then the $k$ entries associated with $i$ in the row indexed by $e$ are given by the vector $\mathbf{a}_{e_{i}}$.

- All other entries are zero.

For example, if $G$ is a 3 -uniform hypergraph, the matrix $\mathbf{M}_{k, 0,3}(G)$ has the following pattern:

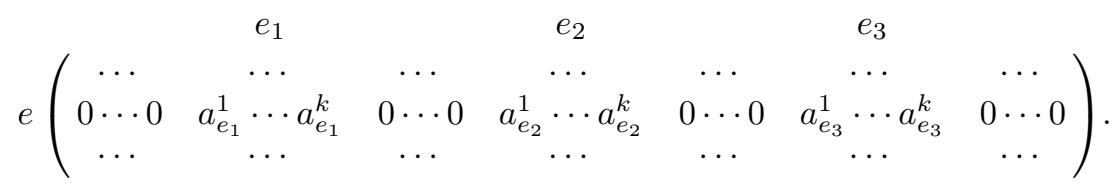

The following lemma is a consequence of the Matroid Union Theorem and a representation result for the $(1,0, d)$-sparsity-matroid due to Edmonds [4]. We give a more direct proof for completeness. We note that Whiteley, in [20, Prop. 2.4], reproduces Edmonds's proof; here, even in the $(1,0, d)$-case we go along different lines.

Lemma 3.1. Let $G$ be a d-uniform hypergraph on $n$ vertices and $m=k n$ edges. Then $\mathbf{M}_{k, 0, d}(G)$ has generic rank $k n$ if and only if $G$ is a $(k, 0, d)$-graph.

Proof. First, we suppose that $G$ is a $(k, 0, d)$-graph. By Lemma 2.2, there is an assignment of a distinct tail to each edge such that each vertex is the tail of exactly $k$ edges. Fix such an orientation, giving a natural association of $k$ edges to each vertex. Now specialize the matrix $\mathbf{M}_{k, 0, d}(G)$ as follows:

- Let $i \in V(G)$ be a vertex that is the tail of edges $e_{i_{1}}, e_{i_{2}}, \ldots, e_{i_{k}}$.

- In row $e_{i_{j}}$, set the variable $a_{e_{i_{j}}}^{j}$ to 1 and all other entries to zero.

Because each edge has exactly one tail, this process defines a setting for the entries of $\mathbf{M}_{k, 0, d}(G)$ with no ambiguity. Moreover, after rearranging the rows and columns, this 
setting of the entries turns $\mathbf{M}_{k, 0, d}(G)$ into the identity matrix, so this example shows its rank generic is $k n$.

In the other direction, we suppose that $G$ is not a $(k, 0, d)$-graph. Since $G$ has $k n$ edges, it is not $(k, 0)$-sparse, so some subgraph $G^{\prime}$ spanning $n^{\prime}$ vertices induces at least $k n^{\prime}+1$ edges. Arranging the edges and vertices of $G^{\prime}$ into the top-left corner of $\mathbf{M}_{k, 0, d}(G)$, we see that $G^{\prime}$ induces a submatrix with at least $k n^{\prime}+1$ rows and only $k n^{\prime}$ columns that are not entirely zero. It follows that $\mathbf{M}_{k, 0, d}(G)$ must be, generically, rank deficient.

Corollary 3.2. The matrix $\mathbf{M}_{k, 0, d}\left(K_{n, d}^{d k}\right)$ is a generic representation for the $(k, 0, d)$ sparsity matroid.

Proof. Lemma 3.1 shows that a $k n \times k n$ matrix minor is generically non-zero if and only if the set of rows it induces corresponds to a $(k, 0, d)$-graph, so the bases of $\mathbf{M}_{k, 0, d}\left(K_{n, d}^{d k}\right)$ are in bijective correspondence with $(k, 0, d)$-graphs.

Corollary 3.3. Let $G$ be a $(k, \ell)$-sparse d-uniform hypergraph with $m$ hyperedges. The set of $\mathbf{v} \in \mathbb{R}^{d k m}$ such that the associated realization of $\mathbf{M}_{k, 0, d}(G)$ has full rank is the open, dense complement of an algebraic subset of $\mathbb{R}^{d k m}$.

Proof. Corollary 3.2 implies that the rank drops only when $\mathbf{v}$ is a common zero of all the $m \times m$ minors of $\mathbf{M}_{k, 0, d}(G)$, which is a polynomial condition.

The natural representation matrix $\mathbf{M}_{k, \ell, d}(G)$. Fix matroidal sparsity parameters $k, \ell$, and $d$, and let $G$ be a $d$-uniform hypergraph. Let $\mathbf{U}$ be an $k n \times \ell$ matrix with generic entries. We define the matrix $\mathbf{M}_{k, \ell, d}(G)$ to be a generic matrix that is a formal solution to the equation (3.1) below, with the entries of $\mathbf{U}$ fixed and the entries of $\mathbf{M}_{k, 0, d}(G)$ as the variables:

$$
\mathbf{M}_{k, 0, d}(G) \mathbf{U}=0
$$

We note that the process of solving (3.1) does not change the location of zero and non-zero entries in $\mathbf{M}_{k, 0, d}(G)$, preserving the naturalness property required by Theorem 1.1.

With this definition, we can restate Theorem 1.1 as follows: the matrix $\mathbf{M}_{k, \ell, d}\left(K_{n, d}^{d k-\ell}\right)$ is a generic representation of the $(k, \ell, d)$-sparsity matroid.

Main lemmas. The next two lemmas give the heart of the proof of Theorem 1.1. The first says that if $G$ is not $(k, \ell)$-sparse, then $\mathbf{M}_{k, \ell, d}(G)$ has a row dependency.

Lemma 3.4. Let $k, \ell$, and $d$ be matroidal parameters and be $G$ a d-uniform hypergraph with $m=k n-\ell$. If $G$ is not $(k, \ell)$-sparse, then $\mathbf{M}_{k, \ell, d}(G)$ is not generically full rank.

Proof. Since $G$ is not $(k, \ell)$-sparse, it must have some vertex-induced subgraph $G^{\prime}$ on $n^{\prime}$ vertices and $m^{\prime}>k n^{\prime}-\ell$ edges. The sub-matrix of $\mathbf{M}_{k, \ell, d}(G)$ induced by the edges of $G^{\prime}$ has at least $k n^{\prime}-\ell+1$ rows and only $k n^{\prime}$ columns that are not all zero, so it must have a row dependency, since, by definition, the kernel of such a sub-matrix has dimension at least $\ell$.

The following is the key lemma. It says that, generically, the dependencies of the type described by Lemma 3.4 are the only ones. 
Lemma 3.5. Let $k, \ell$, and $d$ be matroidal parameters and be $G$ a d-uniform hypergraph with $m=k n-\ell$. If $G$ is $(k, \ell)$-sparse, i.e., it is a $(k, \ell, d)$-graph, then $\mathbf{M}_{k, \ell, d}(G)$ is generically full rank.

Proof. We prove this by constructing an example, from which the generic statement follows. From Corollary 3.2 and Corollary 3.3, we may select values for the variables $a_{e_{i}}^{j}$ in the generic matrix $\mathbf{M}_{k, 0, d}(G)$ so that the resulting realization $\mathbf{M}$ of $\mathbf{M}_{k, 0, d}(G)$ is full rank.

Denote by $\mathbf{m}_{e}$, for $e \in E(G)$, the rows of $\mathbf{M}$. Define the subspace $W_{G}$ of $\mathbb{R}^{k n}$ to be the linear span of the $\mathbf{m}_{e}$. For each vertex-induced subgraph $G^{\prime}$ on $n^{\prime}$ vertices of $G$ define $W_{G^{\prime}}$ to be the linear span of $\left\{\mathbf{m}_{e}: e \in E\left(G^{\prime}\right)\right\} ; W_{G^{\prime}}$ is a subspace of $\mathbb{R}^{k n}$, and, because the $\mathbf{m}_{e}$ span exactly $k n^{\prime}$ non-zero columns in $\mathbf{M}$, it has a natural identification as a subspace of $\mathbb{R}^{k n^{\prime}}$.

We will show that there is a subspace $U$ of $\mathbb{R}^{k n}$ such that $W_{G} \cap U^{\perp}$ has dimension $k n-\ell$; taking the matrix $\mathbf{U}$ to be a basis of $U$ and then solving $\mathbf{m}_{e} \mathbf{U}=0$ for each row of $\mathbf{M}$ gives a solution to (3.1) with full rank. This proves the lemma, since the resulting matrix will have as its rows a basis for $W_{G} \cap U^{\perp}$, which has dimension $k n-\ell$.

Now let $U$ be an $\ell$-dimensional subspace of $\mathbb{R}^{k n}$ with basis given by the columns of the $k n \times \ell$ matrix U. For each vertex-induced subgraph $G^{\prime}$ of $G$ on $n^{\prime}$ vertices, associate the corresponding $k n^{\prime}$ rows of $\mathbf{U}$ to determine a subspace $U_{G^{\prime}}$.

Let $G^{\prime}$ be a vertex-induced subgraph of $G$ on $n^{\prime}$ vertices and consider the subspace $W_{G^{\prime}}$. Since $\operatorname{dim} W_{G^{\prime}}=\operatorname{dim}\left(W_{G^{\prime}} \cap U_{G^{\prime}}\right)+\operatorname{dim}\left(W_{G^{\prime}} \cap U_{G^{\prime}}^{\perp}\right)$, if $\operatorname{dim}\left(W_{G^{\prime}} \cap U_{G^{\prime}}\right)<$ $\operatorname{dim} W_{G^{\prime}}$, then $W_{G^{\prime}} \cap U_{G^{\prime}}$ is at least one-dimensional.

Here is the key to the proof (and where the combinatorial assumption of $(k, \ell)$-sparsity enters in a fundamental way): by the $(k, \ell)$-sparsity of $G$, the dimension of $W_{G^{\prime}}$ is at most $k n^{\prime}-\ell$. Since $U_{G^{\prime}}$ is only (at most) an $\ell$-dimensional subspace of $\mathbb{R}^{k n^{\prime}}$, this only happens if the bases of $W_{G^{\prime}}$ and $U_{G^{\prime}}$ satisfy a polynomial relation. Since there are only finitely many subgraphs, this gives a finite polynomial condition specifying which $U$ are disallowed, completing the proof.

Proof of the Main Theorem 1.1 With the two key Lemmas 3.4 and 3.5, the proof of Theorem 1.1 is very similar to that of Corollary 3.2. We form the generic matrix $\mathbf{M}_{k, \ell, d}\left(K_{n, d}^{d k-\ell}\right)$. Lemma 3.4 and Lemma 3.5 imply that a set of rows forms a basis if and only if the corresponding hypergraph $G$ is a $(k, \ell, d)$-graph.

\section{Extensions: non-uniform hypergraphs and graded sparsity}

In this section, we extend Theorem 1.1 in two directions: to $(k, \ell)$-sparse hypergraphs that are not $d$-uniform; to $(k, \ell)$-graded sparse hypergraphs.

Non-uniform hypergraphs. The theory of $(k, \ell)$-sparsity we developed in [15], does not require that a hypergraph $G$ be $d$-uniform. All the definitions are similar, except we require only that if $\ell \geq(d-1) k$, then each hyperedge have at least $d$ endpoints. The ground set of the corresponding sparsity matroid now is the more complicated hypergraph on $n$ vertices with $i k-\ell$ copies of each hyperedge with $i$ endpoints for $i \geq d$.

The combinatorial properties enumerated in Section 2 all hold in the non-uniform setting, and the proofs in Section 3 all go through verbatim, with slightly more complicated notation, yielding: 
Theorem 4.1 (Natural Realizations: non-uniform version). Let $k$, $\ell$, be integer parameters satisfying the inequality $0 \leq \ell \leq k d-1$. Then, for sufficiently large $n$, the $(k, \ell)$ sparsity-matroid of non-uniform hypergraphs on $n$ vertices is representable by a matrix $\mathbf{M}$ with:

- Real entries

- $k$ columns corresponding to each vertex (for a total of $k n$ )

- One row for each hyperedge e

- In the row corresponding to each edge e, the only non-zero entries appear in columns corresponding to endpoints of $e$

Graded-sparsity. In [10], we developed an extension of $(k, \ell)$-sparsity called $(k, \ell)$ graded-sparsity. Graded-sparsity is the generalization of the sparsity counts appearing in our work on slider-pinning rigidity [16].

Define the hypergraph $K_{n, k}^{+}$, to be the complete hypergraph on $n$ vertices, where hyperedges with $d$ endpoints have multiplicity $d k$. A grading $\left(E_{1}, E_{2}, \ldots, E_{s}\right)$ of $K_{n}^{+}$is a strictly decreasing sequence of sets of edges $E\left(K_{n}^{+}\right)=E_{1} \supsetneq E_{2} \supsetneq \cdots \supsetneq E_{s}$. Now fix a grading on $K_{n}^{+}$and let $G=(V, E)$ be a hypergraph. Define $G_{\geq i}$ as the subgraph of $G$ induced by $E \cap E_{i}$. Let $\ell$ be a vector of $s$ non-negative integers. We say that $G$ is $(k, \ell)$ graded sparse if $G_{\geq i}$ is $\left(k, \ell_{i}\right)$-sparse for every $i$; $G$ is $(k, \ell)$-graded tight if, in addition, it is $\left(k, \ell_{1}\right)$-tight.

The main combinatorial result of [10] is that $(k, \ell)$-graded-sparse hypergraphs form the bases of a matroid, which we define to be the $(k, \ell)$-graded-sparsity matroid.

Theorem 4.2 (Natural Realizations: graded-sparsity). Fix a grading of $K_{n, k}^{+}$and let $k$ and $\ell$ be graded-sparsity parameters. Then, for sufficiently large $n$, the $(k, \ell)$-sparsitymatroid s on $n$ vertices is representable by a matrix $\mathbf{M}$ with:

- Real entries

- $k$ columns corresponding to each vertex (for a total of $k n$ )

- One row for each hyperedge e

- In the row corresponding to each edge e, the only non-zero entries appear in columns corresponding to endpoints of $e$

Because of the presence of the grading, we need to modify the proof of Theorem 1.1 to account for it. The formal matrix $\mathbf{M}_{k, 0,+}\left(K_{n, k}^{+}\right)$is defined analogously to $\mathbf{M}_{k, 0, d}\left(K_{n, d}^{d k-\ell}\right)$, except we sort the rows by the grading. The counterpart to (3.1) then becomes the system:

$$
\mathbf{M}_{k, 0, d}\left(E_{\geq i}\right) \mathbf{U}_{i}=0, i=1,2, \ldots, s
$$

where $V_{1}$ is $k n \times \ell_{1}$, and each successive $\mathbf{U}_{i}$ is $\mathbf{U}_{i}$ with $\ell_{i}$ additional columns.

With this setup, the proof of Theorem 1.1 goes through with appropriate notational changes. 


\section{Conclusions and remarks on rigidity}

We provided linear representations for the matroidal families of $(k, \ell)$-sparse hypergraphs and $(k, \ell)$-graded-sparse hypergraphs that are natural, in the sense that the representing matrices capture the vertex-edge incidence pattern. This family of representations, which extends to the entire matroidal range of sparsity parameters, may be useful as a building block for "Maxwell-Laman-type" rigidity theorems. We conclude with a brief discussion of why one cannot conclude rigidity theorems such as Theorem 1.2 and Theorem 1.3 directly from Theorem 1.1.

The proof of the critical Lemma 3.5 is very general, since it has to work for the entire range of sparsity parameters. What it guarantees is that the entries of $\mathbf{M}_{k, \ell, d}(G)$ are some polynomials, but not what these polynomials are. For rigidity applications, specific polynomials are forced by the geometry, which would require more control over the matrix $\mathbf{U}$ appearing in Equation (3.1) than the proof technique here allows.

For example, in the planar bar-joint rigidity case the "trivial infinitesimal motions" can be given the basis:

- $(1,0,1,0 \ldots, 1,0)$ and $(0,1,0,1, \ldots, 0,1)$, representing infinitesimal translation

- $\left(-y_{1},-y_{2}, \ldots,-y_{n}, x_{1}, x_{2}, \ldots, x_{n}\right)$, representing infinitesimal rotation around the origin

It is important to note that Theorem 1.1 cannot simply be applied with this collection as the columns of $\mathbf{U}$ to conclude the Maxwell-Laman Theorem 1.2. However, using specific properties of the parameters $d=2, k=2, \ell=3$ Lovász and Yemini [12] do prove the Maxwell-Laman Theorem starting from an algebraic result in the same vein as our Lemma 3.5 , providing evidence that our results may have some relevance to rigidity.

\section{References}

[1] M. O. Albertson and R. Haas, Bounding functions and rigid graphs. SIAM J. Discrete Math. 9 (1996), 269-273.

[2] T. Brylawski, Constructions, in N. White (ed.), Theory of Matroids, Encyclopedia of Mathematics and Its Applications, chapter 7, Cambridge University Press, 1986, 127-223.

[3] D. A. Cox, J. Little and D. O'Shea, Ideals, Varieties and Algorithms, Undergraduate texts in Mathematics, Springer Verlag, New York, second edition, 1997.

[4] J. Edmonds, Systems of distinct representatives and linear algebra, J. Res. Nat. Bur. Standards Sect. B, 71 (1967), 241-245.

[5] R. Haas, A. Lee, I. Streinu and L. Theran, Characterizing sparse graphs by map decompositions, J. Combin. Math. Combin. Comput. 62 (2007), 3-11.

[6] N. Katoh and S. Tanigawa, A proof of the molecular conjecture, in Proc. 25th Symp. on Computational Geometry (SoCG'09), 2009, 296-305.

[7] G. Laman, On graphs and rigidity of plane skeletal structures, J. Eng. Math. 4 (1970), 331-340.

[8] A. Lee, Geometric Constraint Systems with Applications in CAD and Biology, PhD thesis, University of Massachusetts Amherst, May 2008.

[9] A. Lee and I. Streinu, Pebble game algorithms and sparse graphs, Discrete Math. 308 (2008), $1425-1437$.

[10] A. Lee, I. Streinu and L. Theran, Graded sparse graphs and matroids, J. Univers. Comput. Sci. 13 (2007), 1671-1679. 
[11] M. Loréa, On matroidal families, Discrete Math. 28 (1979), 103-106.

[12] L. Lovász and Y. Yemini, On generic rigidity in the plane, SIAM J. Algebra. Discr. 3 (1982), 91-98.

[13] J. Maxwell, On the calculation of the equilibrium and stiffness of frames, Philosophical Magazine Series 4, Jan 1864.

[14] I. Streinu and L. Theran, Sparsity-certifying graph decompositions, Graph. Combinator. 25 (2009), 219-238.

[15] I. Streinu and L. Theran, Sparse hypergraphs and pebble game algorithms, Eur. J. Combin. 30 (2009), 1944-1964.

[16] I. Streinu and L. Theran, Slider-pinning rigidity: a Maxwell-Laman-type theorem, Discrete Comput. Geom. 44 (2010), 812-837.

[17] T.-S. Tay, Rigidity of multigraphs I: linking rigid bodies in $n$-space, J. Comb. Theory B 26 (1984), 95-112.

[18] N. White and W. Whiteley, The algebraic geometry of motions of bar-and-body frameworks, SIAM J. Algebra. Discr. 8 (1987), 1-32.

[19] W. Whiteley, The union of matroids and the rigidity of frameworks, SIAM J. Discrete Math. 1 (1988), 237-255.

[20] W. Whiteley, A matroid on hypergraphs, with applications in scene analysis and geometry, Discrete Comput. Geom. 4 (1989), 75-95.

[21] W. Whiteley, Some matroids from discrete applied geometry, in: J. Bonin, J. G. Oxley, and B. Servatius (eds), Matroid Theory, volume 197 of Contemp. Math. (1996), 171-311. 